\title{
Frustrated kinetic energy, the optical sum rule, and the mechanism of superconductivity
}

\author{
Sudip Chakravarty ${ }^{1}$, Hae-Young Kee ${ }^{1}$, and Elihu Abrahams ${ }^{2}$ \\ ${ }^{1}$ Department of Physics and Astronomy, University of California Los Angeles \\ Los Angeles, CA 90095-1547 \\ ${ }^{2}$ Serin Physics Laboratory, Rutgers University, Piscataway, NJ 08854-8019
}

(June 14, 2022)

\begin{abstract}
The theory that the change of the electronic kinetic energy in a direction perpendicular to the CuOplanes in high-temperature superconductors is a substantial fraction of the condensation energy is examined. It is argued that the consequences of this theory based on a rigorous $c$-axis conductivity sum rule are consistent with recent optical and penetration depth measurements.
\end{abstract}

\section{PACS:}

a. Introduction. The aim of this Letter is to partly resolve a number of issues [1] 9] related to a theory of high-temperature superconductivity known as the interlayer tunneling theory (ILT) [10] and to propose the efficacy of a conductivity sum rule. Within a simple version of ILT, one relates the zero-temperature $c$-axis penetration depth $\lambda_{c}$ to the superconducting condensation energy [1]. Here, we point out that the realization of ILT and the interpretation of recent measurements of $\lambda_{c}$ [2,3], necessarily require more careful analysis and that the two can be brought into agreement. In addition, we argue that ILT accounts for two features of $c$-axis optical measurements: (1) the observation that the $c$-axis (perpendicular to the $\mathrm{CuO}$-planes) kinetic energy is substantially reduced in the superconducting state [5] and (2) the correlation ("Basov correlation") between $\lambda_{c}$ and the $c$-axis conductivity in the normal state [11.

For our purposes, the content of ILT is that a significant portion of the superconducting condensation energy comes from the change in the $c$-axis kinetic energy as the electrons enter the superconducting state. It is a phenomenological fact that this kinetic energy is frustrated in the normal state, but that the frustration is relieved in the superconducting state as the coherent tunneling of pairs becomes possible, resulting in a sharp plasma edge in the reflectivity 12 .

Recently, Anderson [1] conjectured that the full condensation energy is derived from the $c$-axis Josephson energy, which, in turn, determines the penetration depth. Then, using estimates of the condensation energies, he predicted $\lambda_{c}$. On the basis of recent experiments [2,3], it has been suggested that this prediction is strongly violated in both $\mathrm{Tl} 2201$ and $\mathrm{Hg}$ 1201, although it appears to hold for LSCO for a large range of doping. The singlelayer superconductors containing one $\mathrm{CuO}$-plane per unit cell are emphasized because they pose the most stringent test of ILT.

However, the situation is not so clear. (1) As shown earlier [7], the predicted $\lambda_{c}$ should be a factor 2 larger than that predicted in [1]. (2) The measured values of $\lambda_{c}$ are in disagreement. Vortex imaging measurements
(2) give $\lambda_{c}=17-19 \mu \mathrm{m}$ in $\mathrm{Tl} 2201$, while it is $12 \mu \mathrm{m}$ in the optical measurements [5], in a similar sample. For $\mathrm{Hg}$ 1201, vortex measurements give $8 \mu \mathrm{m}$ [3], while the optical measurements give $6.2 \mu \mathrm{m}$, again in a similar sample [13], and, disturbingly, magnetic measurements yield $1.36 \mu \mathrm{m}$ 14. (3) The normal state electronic specific heat must be extrapolated to $T=0$ from above $T_{c}$ to determine the condensation energy.

There is an even more fundamental difficulty. The condensation energy is well-defined only within mean field theory. For those materials that deviate from mean field behavior, that is, those that do not have a sharp specific heat jump at $T_{c}$, the condensation energy cannot be determined by a simple integration of the specific heat [1]. The electronic specific heat in Tl 2201 [15 and in $\mathrm{Hg} 1201$ [16] show large fluctuation effects; we shall show that in these cases, agreement with experiments can be achieved if the fluctuation effects are subtracted out.

b. Sum rule. We now discuss the $c$-axis conductivity sum rule [7]. Consider the full hamiltonian $H=H_{\text {rest }}+$ $H_{c}$; the $c$-axis kinetic energy is defined by

$$
H_{c}=-\sum_{i j, s} t_{\perp}(i j, l) c_{i l, s}^{\dagger} c_{j l+1, s}+\text { h.c.; }
$$

the remainder, $H_{\text {rest }}$, contains no interplane interaction terms 17], but it is otherwise arbitrary and may contain impurity interactions that couple to the charge density. The hopping matrix element $t_{\perp}(i j, l)$, where $(i, j)$ refer to the sites of the two-dimensional lattice, and $l$ to the layer index, can be random in the presence of impurities [18. The electron operators $\left(c, c^{\dagger}\right)$ are also labeled by a spin index $s$. We denote the magnitude of $t_{\perp}(i j, l)$ by $t_{\perp}$. It is easy to adapt a sum rule derived first by Kubo 19. to get a sum rule for the $c$-axis optical conductivity $\sigma^{c}(\omega, T)$, which is

$$
\int_{0}^{\infty} d \omega \operatorname{Re} \sigma^{c}(\omega, T)=\frac{\pi e^{2} d^{2}}{2 \hbar^{2}(A d)}\left\langle-H_{c}\right\rangle .
$$

Here the average refers to the quantum statistical average, $A$ to the two-dimensional area, and $d$ to the separation between the $\mathrm{CuO}$ planes. 
The hamiltonian $H_{c}$ is an effective hamiltonian valid for low energy processes that do not involve interband transitions. It can be derived by a downfolding process, in which all the higher energy bands are integrated out 21. Because interband processes involve large energy differences, a second order downfolding procedure is sufficient. This is essentially how one derives the effective mass contribution of higher energy bands.

Since $H_{c}$ is a low-energy effective hamiltonian, the upper limit in Eq. (2) can not exceed an interband cutoff $\omega_{c}$, of order $2-3 \mathrm{eV}$. In the superconducting state, $\sigma^{c s}(\omega, T)=D_{c}(T) \delta(\omega)+\sigma_{\text {reg }}^{c s}(\omega, T)$, where $D_{c}(T)$ is the superfluid weight. From Eq. (2), it follows that

$$
\begin{aligned}
D_{c}\left(T_{1}\right) & =\int_{0^{+}}^{\omega_{c}} d \omega\left[\operatorname{Re} \sigma^{c n}\left(\omega, T_{2}\right)-\operatorname{Re} \sigma_{\mathrm{reg}}^{c s}\left(\omega, T_{1}\right)\right] \\
& +\frac{\pi e^{2} d^{2}}{2 A d \hbar^{2}}\left[\left\langle-H_{c}\left(T_{1}\right)\right\rangle_{s}-\left\langle-H_{c}\left(T_{2}\right)\right\rangle_{n}\right]
\end{aligned}
$$

Here, if $T_{2}<T_{c},\left\langle-H_{c}\left(T_{2}\right)\right\rangle_{n}$ is to be understood as taken in the normal state extrapolated to below $T_{c}$, and $\sigma^{c n}$ is the corresponding conductivity.

c. Lowering of the kinetic energy in the superconducting state: Basov et al. [5] have tested the sum rule, Eq. (3), by setting $T_{1} \ll T_{c}$ and $T_{2}=T_{c}$. The result is that up to $0.15 \mathrm{eV}$, the integral over the conductivity is only half the left hand side, so that the remaining half must then be the change in the $c$-axis kinetic energy of electrons. Since we expect the normal state kinetic energy to become less negative as the temperature is lowered [20], this measured change of the kinetic energy is only a lower bound on the difference in the kinetic energy at $T=0$. Thus, these experiments support the fundamental statement of ILT that the $c$-axis kinetic energy is substantially lowered in the superconducting state, in contrast to BCS.

d. The $T=0$ superfluid weight $D_{c}$ and $\lambda_{c}$ : We relate $\lambda_{c}$ to the change in $c$-axis kinetic energy as follows: We set $T_{1}=T_{2}=0$ in Eq. (3). From the experiments of Ando et al. 20], it is seen that the $c$-axis response in the normal state obtained by destroying superconductivity is insulating as $T \rightarrow 0$; it follows that $\sigma^{c n}(\omega, T=0) \sim \omega^{2}$, as $\omega \rightarrow 0$. The regular part $\sigma_{\text {reg }}^{c s}(\omega, T=0)$ is also expected to vanish as a power law in a $d$-wave superconductor 27]. At high frequencies the two conductivities must, however, approach each other. Consequently it is reasonable to hypothesize that the conductivity integral on the right hand side of Eq. (3) is negligibly small. Therefore,

$$
D_{c}(0)=\frac{c^{2}}{8 \lambda_{c}^{2}} \approx \frac{\pi e^{2} d^{2}}{2 A d \hbar^{2}}\left[\left\langle-H_{c}\right\rangle_{s}-\left\langle-H_{c}\right\rangle_{n}\right]
$$

where we assumed local London electrodynamics. We emphasize that the choice of the normal state in Eq. (4) is not arbitrary because we have assumed that the integral on the right hand side of Eq. (3) is vanishingly small, and this would not be true for an arbitrary state. In any case, the right hand side should be a lower bound.

e. Condensation energy: The attempt to extract the condensation energy from the specific heat data runs into ambiguity, except within a mean field treatment. In the presence of fluctuations, superconducting correlations, which can primarily be of in-plane origin, contribute to the energy and significantly to the specific heat of the normal state. We suggest that this is indeed the case for Tl 2201 (see below), for example. To resolve this ambiguity, instead of the conjecture made by Anderson [1], we propose to subtract the fluctuation effects and to use the remainder as an effective specific heat from which to extract the $c$-axis contribution to the condensation energy. The rationale is that free energy can be decomposed into a singular and a non-singular part. The universal singular part is more sensitive to collective long-wavelength fluctuations, while the non-singular part is dominated by short distance microscopic pairing correlations. This procedure is well suited to ILT, because, in this theory, the effective "mean field" condensation energy can be enhanced due to pair tunneling between layers [7]. Note that there is no simple relation between $T_{c}$ and condensation energy, except in mean field theory.

The fit to the specific heat of $\mathrm{Tl} 2201$ to 2D Gaussian fluctuation plus non-singular terms 29.30 is shown in Fig. 11. We have used $C\left(T>T_{c}\right)=\gamma T+g_{+} / t$

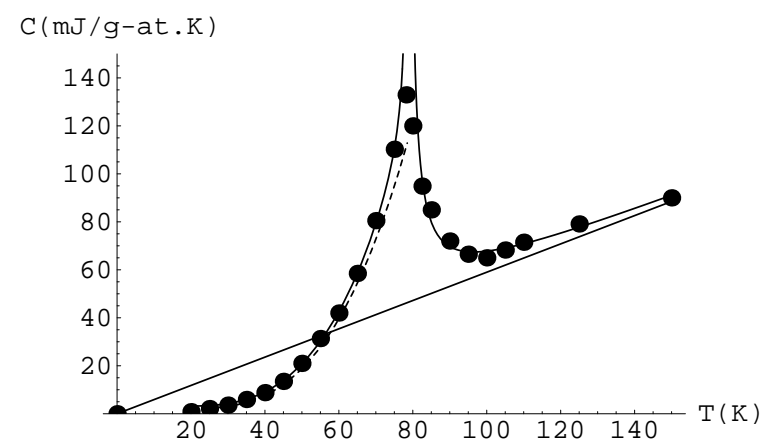

FIG. 1. The electronic specific heat data of $\mathrm{Tl} 2201$ [15] fitted to a combination of singular and analytic terms (solid line); $T_{c}=78.7 \mathrm{~K}$. The straightline is $\gamma T$, and the dashed line is the analytic part of the specific heat below $T_{c}$.

and $C\left(T<T_{c}\right)=c_{0} T\left(1+c_{1} t+c_{2} t^{2}\right)+g_{-} / t$, where $t=\left|1-T / T_{c}\right|\left(\gamma=0.59, g_{+}=2.38, g_{-}=0.74, c_{0}=\right.$ $\left.1.44, c_{1}=-2.79, c_{2}=2.07\right)$. The fit for 3D Gaussian fluctuations is considerably worse. This suggests that the in-plane contributions dominate. The data for $\mathrm{Hg} 1201$ are too imprecise to do the same, but clearly fluctuation effects are quite prominent and a proper subtraction should result in a larger prediction for $\lambda_{c}$. Optimally doped LSCO does not exhibit fluctuation effects that are as pronounced. For underdoped LSCO, we were unable to use the specific heat data [15] as they do not seem to 
fit any simple form.

Condensation energies are obtained from an integration of the measured specific heat. In Table t we show both results with $\left(\Delta E_{\mathrm{sub}}\right)$ and without $\left(\Delta E_{0}\right)$ subtracting fluctuation effects. By using these values for the right hand side of Eq. (化) we extract the corresponding values of $\lambda_{c}$ as shown along with the experimental values [22]. The precision of the present Tl 2201 specific heat data is

\begin{tabular}{c|c|c|c}
\hline \hline & LSCO (15\%) & Tl 2201 & Hg 1201 \\
\hline$\Delta E_{0}$ & $\sim 150$ & $\sim 825$ & $\sim 850$ \\
$\Delta E_{\text {sub }}$ & - & $\sim 25$ & - \\
$\lambda_{c, 0}$ & $\sim 6.5$ & $\sim 1.7$ & $\sim 2$ \\
$\lambda_{c, \text { sub }}$ & - & $\sim 10$ & - \\
$\lambda_{c, \text { exp }}$ & $5.5[28]$ & $12-19[2,5]$ & $6-8[3,12]$ \\
\hline \hline
\end{tabular}

TABLE I. Condensation energies (in $\mathrm{mJ} / \mathrm{g}$-at) and penetration depths (in $\mu \mathrm{m}$ ). Precise error estimates are unavailable.

not sufficient to make a precise subtraction of the fluctuation contribution. The uncertainty is considerable; a reasonable guess for the uncertainty in $\Delta E_{\text {sub }}$ is $25 \pm 15$. Nonetheless, it is clear that using the subtracted value gives a much larger penetration depth.

f. The Basov correlation: We manipulate the right hand side of Eq. (2) to draw further conclusions. We perform a canonical transformation such that $H_{c}$ is eliminated from the hamiltonian $H$. Thus,

$$
\tilde{H}=e^{-S} H e^{S}=H_{\text {rest }}+\frac{1}{2}\left[H_{c}, S\right]+\cdots,
$$

where the antihermitian operator $S$ is defined by $H_{c}+$ $\left[H_{\text {rest }}, S\right]=0$. The ground state $|\tilde{0}\rangle$ of the full hamiltonian $H$ can be determined perturbatively in $S$ (or, equivalently $t_{\perp}$ ) to show that the ground state expectation value of the $H_{c}$ is given by

$$
\left\langle\tilde{0}\left|H_{c}\right| \tilde{0}\right\rangle=-2 \sum_{n \neq 0} \frac{\left|\left\langle 0\left|H_{c}\right| n\right\rangle\right|^{2}}{E_{n}-E_{0}}+O\left(t_{\perp}^{4}\right),
$$

where $E_{n}$ and $|n\rangle$ are the eigenvalues and eigenfunctions of $H_{\text {rest }}$. Of course, the same result could be obtained directly without making a canonical transformation. We have taken this route to hint that the canonical transformation, if carried out in infinitesimal steps, could potentially be a powerful method to obtain the effective low-energy hamiltonian 23].

For conserved parallel momentum, the expansion on the right hand side of Eq. (5) does not converge in a Fermi liquid theory because of vanishing energy denominators; therefore the expansion would not be valid. In a gapped state, the expansion can be legitimate because of the absence of vanishing energy denominators. In a non-Fermi liquid state, the matrix elements should vanish for vanishing energy differences, and the the sum is skewed to high energies. Thus, the energy denominator can be approximated by $W$ [24], and the sum can be collapsed using the completeness condition to $\left\langle\tilde{0}\left|H_{c}\right| \tilde{0}\right\rangle \approx-\left\langle 0\left|H_{c}^{2}\right| 0\right\rangle / W$. The effective hamiltonian $-H_{c}^{2} / W$ is identical to the hamiltonian of previous realizations of ILT [25, 26].

Thus $\left\langle H_{c}\right\rangle$ is of order $t_{\perp}^{2} / W$. Then, from Eq. (2) for example, one can see that on dimensional grounds the $c$-axis conductivity is

$$
\sigma_{c}(T)=a\left(\frac{e^{2} d t_{\perp}^{2}}{A W \hbar^{2}}\right) \frac{1}{\Omega(T)},
$$

where $a$ is a numerical constant weakly dependent on the band structure. The inelastic scattering rate is proportional to the unknown function $\Omega(T)$. Combining the result of the previous paragraph with Eqs. (4.,7), we find

$$
\frac{c^{2}}{\lambda_{c}^{2}}=\frac{4 \pi}{a} \sigma_{c}(T) \Omega(T)\left[u_{s}-u_{n}\right],
$$

where $u_{s, n}$ is $\left\langle 0\left|\left(H_{c} / t_{\perp}\right)^{2}\right| 0\right\rangle_{s, n}$. The average here is with respect to the ground state of $H_{\text {rest }},|0\rangle$ not $|\tilde{0}\rangle$. The product $\sigma_{c}(T) \Omega(T)$ is independent of $T$.

For underdoped to optimally doped materials, the $c$ axis resistivity, $\rho_{c}(T)$, can often be fitted to [31]

$$
\rho_{c}(T)=b_{1} T^{-p}+b_{2}^{\prime} T .
$$

The logarithmic behavior [20] obtains in the limit $p \rightarrow 0$. If we express Eq. (8) in terms of the temperature $T^{*}$ at which the resistivity takes its minimum value, we get

$$
\frac{c^{2}}{\lambda_{c}^{2}}=4 \pi \sigma_{c}\left(T^{*}\right) T^{*}\left\{\frac{b_{2}(p+1)}{p}\left[u_{s}-u_{n}\right]\right\},
$$

where $b_{2}=b_{2}^{\prime}\left(d e^{2} t_{\perp}^{2} / \hbar^{2} A W\right)$. The expression in the curly brackets depends dominantly on $b_{2}$, which describes the high temperature resisitivity. The low temperature behavior enters only through the exponent $p$. Thus, provided the expression in curly brackets is a universal constant, a plot of $\ln \lambda_{c}$ against $\ln \left[\sigma_{c}\left(T^{*}\right) T^{*}\right]$ should be a universal straight line, independent of material, with a slope of $-1 / 2$. Basov et al. [11] discovered a similar correlation by plotting $\ln \lambda_{c}$ against $\sigma_{c}\left(T_{c}\right)$, shown as (I) of Fig. 2. The correlation discussed here, shown as (II), is excellent. The data for underdoped LSCO, however, are affected by both the structural transition and the (1/8)anomaly. In Fig. 2, we have taken $T^{*} \approx T_{c}$ for those optimally doped materials that show simply a flattening of $\rho(T)$ close to $T_{c}$. Thus, we see that $b_{2}$ is indeed inversely proportional to $\left(u_{s}-u_{n}\right)$, which, in ILT, is proportional to the $T=0$ superfluid density, $n_{s}(0)$. This can be tested further in future experiments 33].

g. Conclusion: ILT accounts for a number of experimental behaviors, in particular the Basov correlation, and it provides a recipe for determining the $c$-axis penetration depth. In Tl 2201 and $\mathrm{Hg} 1201$ there must be 


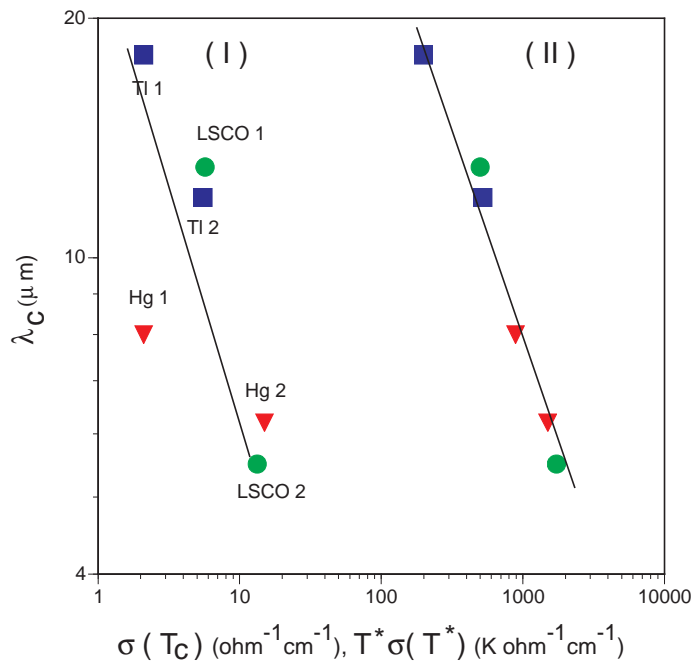

FIG. 2. The Basov plot: $\ln \lambda_{c}$ is plotted against $\ln \sigma_{c}\left(T_{c}\right)$, as in the original Basov plot (I), and against $\ln \left[T^{*} \sigma_{c}\left(T^{*}\right)\right]$ as discussed here (II). The legends in group (II) are the same as those in group (I). Tl1: Ref. [2]; Tl2: Ref. [5]; Hg1: Ref. [3], Hg2: Ref. [13]; LSCO 1 (12\%), LSCO 2 (15\%): Refs. [15, 28, $32]$.

strong superconducting correlations in the normal state. The source of these must be both the fluctuation effects not contained in the mean field treatment of ILT as well as substantial in-plane pairing correlations. Although ILT is not the main driving mechanism for Tl 2201, it may be for LSCO and in any case ILT remains an important mechanism which can enhance $T_{c}$ in both single layer and multilayer materials [7, 19,25].

We thank P. W. Anderson for extensive discussions. The experiments of D. Basov, K. A. Moler and their coworkers have been instrumental in sharpening our thoughts and we thank them for many discussions. E. A. and S. C. are supported by grants from the National Science Foundation. H. -Y. Kee is supported by a Collaborative UC/Los Alamos Research grant.

[1] P. W. Anderson, Science 279, 1196 (1998).

[2] K. A. Moler et al., Science 279, 1193 (1998).

[3] J. R. Kirtley et al., Phys. Rev. Lett. 81, 2140 (1998).

[4] A. J. Leggett, Science 274, 587 (1996).

[5] D. Basov, et al., Science, in press.

[6] B. Farid, J. Phys. Condensed Matter 10, L589 (1998), and preprint.

[7] S. Chakravarty, Euro. Phys. J. B 5, 337 (1998); condmat/9801025

[8] J. Schützmann et al., Phys. Rev. B 55, 11118 (1997).

[9] A. A. Tvestkov et al., Nature 395, 360 (1998).

[10] P. W. Anderson, The Theory of Superconductivity in
High-T $T_{c}$ Cuprates (Princeton University Press, Princeton, 1997).

[11] D. Basov, et al., Phys. Rev. B 50, 3511 (1994).

[12] S. Uchida et al., Phys. Rev. Lett. 69, 1445 (1992).

[13] For a sample $\mathrm{Hg} 1201$ of $T_{c}=96 \mathrm{~K}, \lambda_{c}=6.2 \mu \mathrm{m}, \mathrm{D}$. Basov et al., to be published.

[14] C. Panagopoulos et al., Phys. Rev. Lett. 79, 2320 (1997); T. Xiang, C. Panagopoulos and J. R. Cooper, condmat/9807404.

[15] J. W. Loram et al., Physica C 235, 134 (1994).

[16] B. Billon et al., Phys. Rev. B 56, 10824 (1997).

[17] The sum rule remains unchanged if interplane interactions that do not transfer electrons between the planes are allowed.

[18] The generalization to further than nearest neighbor hopping is contained in the paper by Kubo [19].

[19] R. Kubo, J. Phys. Soc. Japan 12, 570 (1957). See also, B. S. Shastry and Bill Sutherland, Phys. Rev. Lett. 65, 243 (1990); D. J. Scalapino, S. R. White, and S. C. Zhang, Phys. Rev. B 47, 7995 (1993); J. E. Hirsch, Physica C 199, 305 (1992); E. Kim, cond-mat/971229.

[20] Y. Ando et al., Phys. Rev. Lett. 75, 4662 (1995); G. S. Boebinger et al., Phys. Rev. Lett. 77, 5417 (1996).

[21] O. K. Andersen et al., J. Phys. Chem. Solids 56, 1573 (1995).

[22] The statement that the experimental penetration depth for the optimally doped LSCO is $3 \mu \mathrm{m}$ 便 is erroneous. The estimates of the condensation energy are also incorrect in this paper.

[23] S. D. Glazek and K. G. Wilson, Phys. Rev. D 48, 5863 (1993); ibid 49, 4214 (1994); F. Wegner, Ann. Physik 3, 77 (1994).

[24] The difference between $\int_{0}^{W} d \omega \omega^{2 \alpha} / \omega$ and $\int_{0}^{W} d \omega \omega^{2 \alpha} / W$ is an unimportant numerical factor that can be absorbed in the definition of the cutoff $W$. A similar conclusion was reached by A. G. Abanov and P. Wiegmann, Phys. Rev. Lett. 78, 4103 (1997).

[25] S. Chakravarty, A. Sudbø, P. W. Anderson, and S. Strong, Science 261, 337 (1993).

[26] S. Chakravarty and P. W. Anderson, Phys. Rev. Lett. 72, 3859 (1994).

[27] A. Hosseini et al., Phys. Rev. Lett. 81, 1298 (1998).

[28] S. Uchida et al. Phys. Rev. B 53, 14558 (1996); T. Shibauchi et al., Phys. Rev. Lett. 72, 2263 (1994); see also Ref. 朐.

[29] S. E. Inderhees et al., Phys. Rev. Lett. 60, 1178 (1988).

[30] L. N. Bulaevski, V. L. Ginzburg, and A. A. Sobyanin, Physica C 152, 378 (1988); ibid C 156, 652 (1988); K. F. Quader and E. Abrahams, Phys. Rev. 38, 11977 (1988).

[31] The conclusion is unchanged if we consider a more general expression, $\rho_{c}(T)=b_{1} T^{-p}+b_{2} T^{q}$.

[32] Y. Nakamura and S. Uchida, Phys. Rev. B 47, 8369 (1993).

[33] The relation $b_{2} \propto 1 / n_{s}(0)$ can be made plausible if one considers scattering from collective phase modes [V. J. Emery and S. A. Kivelson, Nature 374, 434 (1995).] whose stiffness is proportional to $n_{s}(0)$ (H.-Y. Kee, Y.-B. Kim, S. Chakravarty, and E. Abrahams, unpublished.) 